\title{
Erratum
}

\section{Genome-Wide Association Study of Alcohol Dependence Implicates KIAA0040 on Chromosome Iq}

Lingjun Zuo, Joel Gelernter, Clarence K Zhang, Hongyu Zhao, Lingeng Lu, Henry R Kranzler, Robert T Malison, Chiang-Shan R Li, Fei Wang, Xiang-Yang Zhang, Hong-Wen Deng, John H Krystal, Fengyu Zhang and Xingguang Luo

Neuropsychopharmacology (20 I2) 37, 58 I-582; doi: | 0.1038/npp.20 I I.27 |

Correction to: Neuropsychopharmacology advance online publication, 28 September 2011; doi:10.1038/npp.2011.229
In this article, the incorrect version of Figure 1 was published. The correct version is shown in the next page. 
a

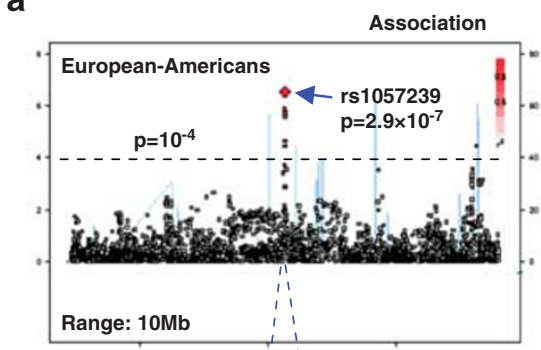

b
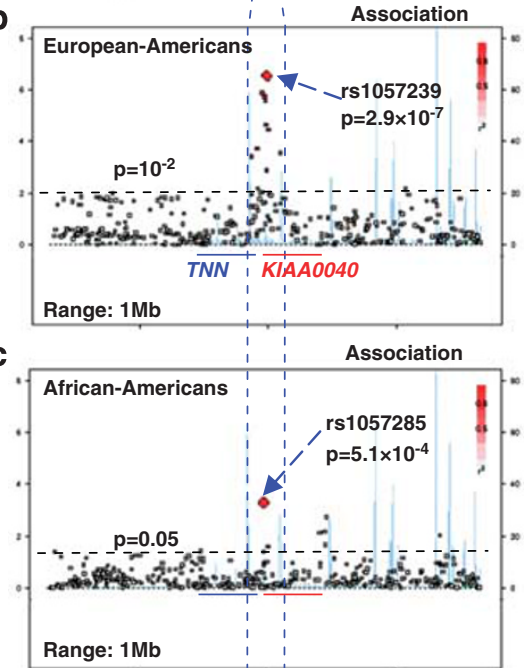

d

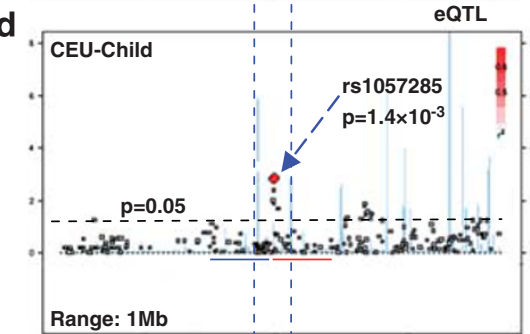

e
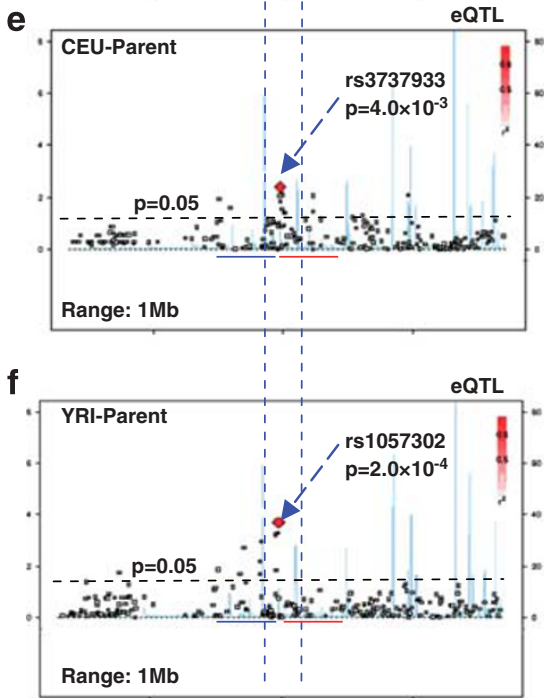

g

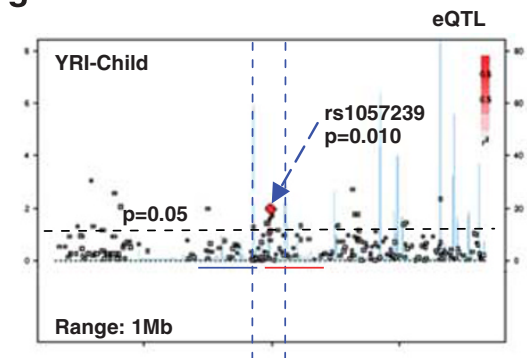

$\mathbf{h}$
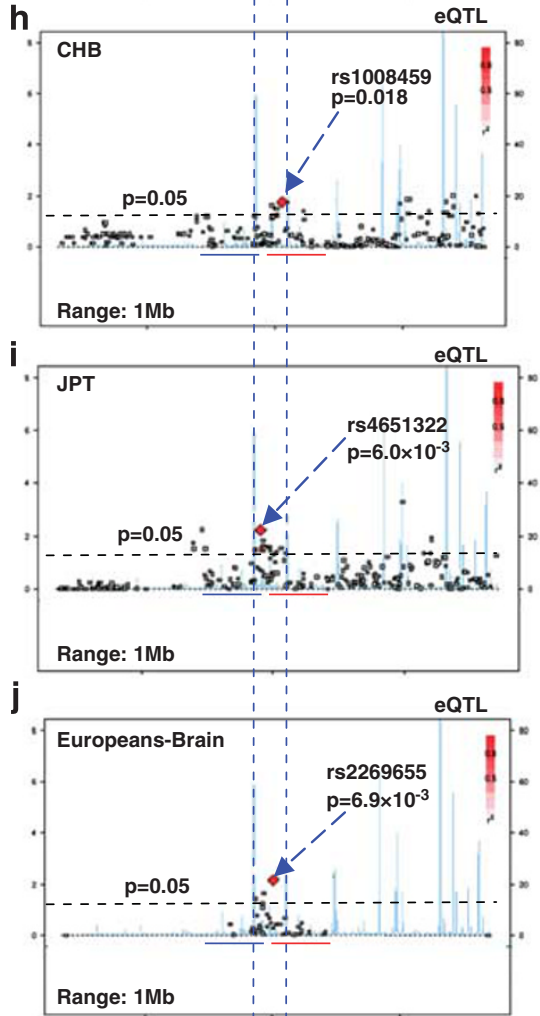

k
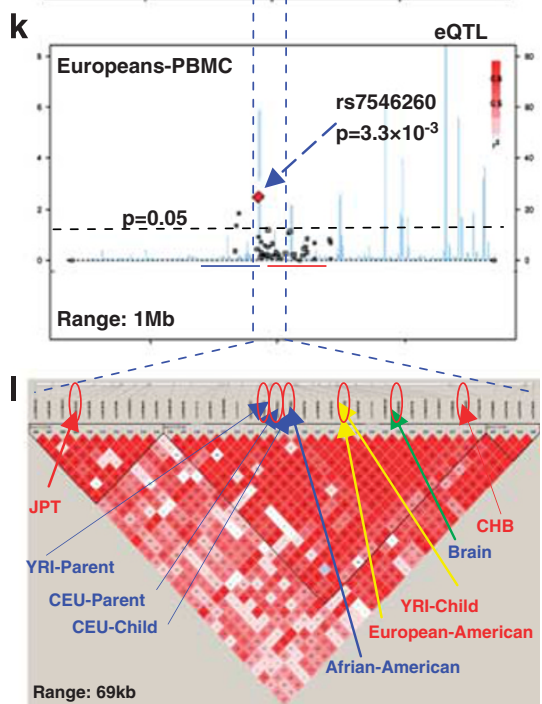